\title{
The Research on Analysis of Effectiveness and Cost in Application of Turboshaft Engine Demonstration and Decision
}

\author{
Yifan Wang ${ }^{1, a}$, Shiyu Gong ${ }^{1, b}$ and Bohua $\mathrm{Li}^{1, \mathrm{c}}$ \\ ${ }^{1}$ College of Information System and Management, National University of Defense Technology, \\ Changsha 410073, China; \\ a349563505@qq.com, byiyue@189.cn, 'Ibhwindy@outlook.com
}

Keywords: Turboshaft Engine, Demonstration, Analysis of Effectiveness and Cost, Decision.

\begin{abstract}
At present, the scientific and detailed process is lacking on the demonstration of turboshaft engine, and the demonstration stage is very important for the development of new pattern turboshaft engine. Practices show that it has made up 80 percent of the life cycle cost at the end of demonstration, and the cost of demonstration is only 3 percent of the life cycle cost. The analysis of effectiveness and cost is decision-making method about handling complex multiple factors, optimizing multi-objective and multi-scheme, it can effectively regulate the life cycle cost by applying the analysis of effectiveness and cost in demonstration. This paper makes extensively study on the demonstration process, which reveals the effects and methods on demonstration and decision-making process by the analysis of effectiveness and cost. These researches will be of great importance in enhancing the scientific validity of the demonstration of turboshaft engine.
\end{abstract}

\section{Introduction}

The demonstration of turboshaft engine is complex decision-making process involved multi-objective, multi-factor and multi-scheme, which result has crucial effect on achieving development target in subsequent engineering project. The analysis of effectiveness and cost is a decision-making method for handling complex multiple factors, optimizing multi-objective and multi-scheme. At present, there exists a lack of intensive research about applying analysis of effectiveness and cost to decision in demonstration of weapons and equipments involved turboshaft engine. This paper makes extensively study on the decision-making process of the demonstration of turboshaft engine, which reveals these various needs of decision on different link involved multi-objective, multi-factor and multi-scheme. Then, accounts targeted for the function on how to solve these decision-making problem about analysis of effectiveness and cost, puts up the ways on carrying out research about analysis of effectiveness and cost. These researches will be of great importance in enhancing the scientific validity of the demonstration of turboshaft engine.

\section{The Analysis of demonstration and decision-making process of turboshaft engine}

The demonstration of weapons and equipments is a optimization procedure according to the future usage requirement of weapons and equipments, it based on the present technology, budget, development time and other constrains, by means of developing new equipments or improve the existing ones to research a relatively satisfied solution[1]. For the new pattern turboshaft engine, its demonstration procedure include requirement demonstration, performance demonstration, comprehensive model system demonstration, tactical technique index demonstration and scheme evaluation, as shown in the figure 1. 


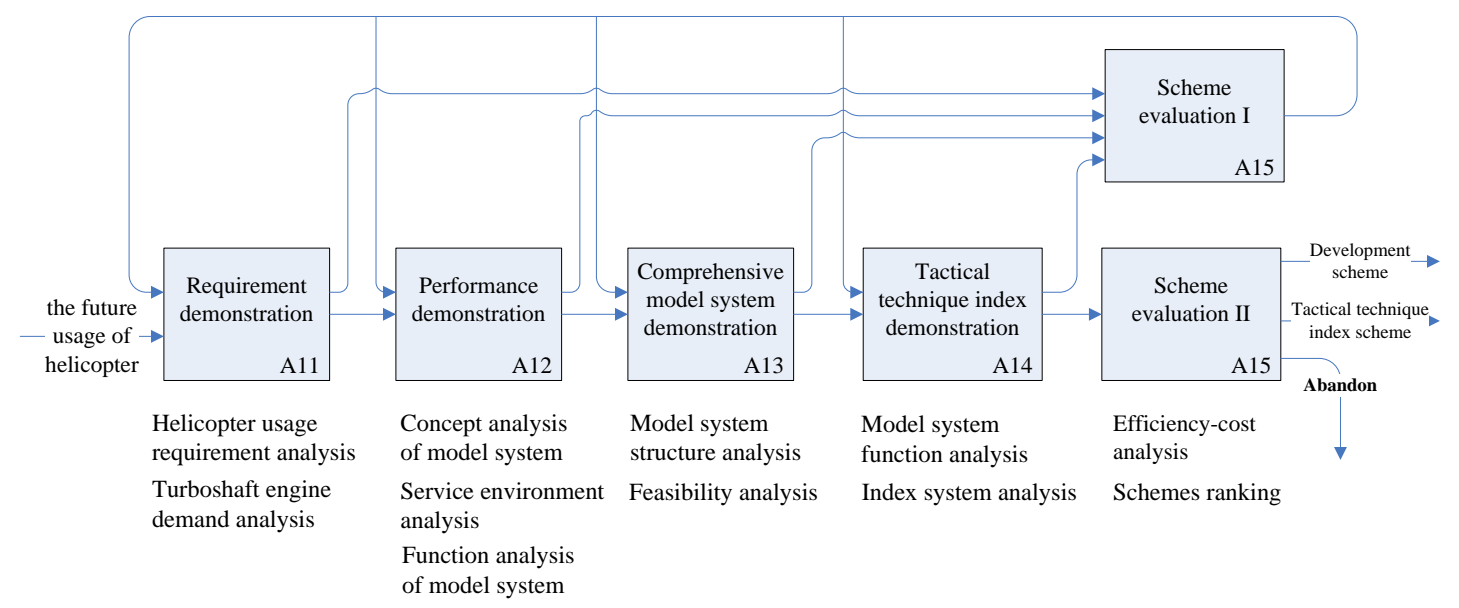

Fig. 1. Demonstration Procedure of Turboshaft Engine

The requirement demonstration composes of two sub-procedures: helicopter usage requirement analysis and turboshaft engine demand analysis, which used to transform the usage requirement into ability demanded. Under the helicopter usage requirement analysis, the missions of turboshaft engine from the future usage of helicopter is decomposed into series of submissions, and the homologous mission index is built at the same time. Through the mapping relation between mission index with ability indicator, the usage requirement is transformed into ability demanded which is described by ability indicator. Various kinds of submissions and their combinations can be mapped to various ability demanded combinations. So, the ability demanded scheme of turboshaft engine is faced with various possible choices; the best ability demanded scheme should be made under the foundation of estimation and decision. Namely, above processes are called development demanded demonstration and decision-making link of turboshaft engine. Later, the turboshaft engine demand analysis discussed whether the development of turboshaft engine should proceed to fulfill the future usage of helicopter by analyzing the development situation of turboshaft engine. There are two different development schemes in this sub-procedure, one is directly improving the existing engines, another is developing new pattern turboshaft engine. That is the development approach demonstration and decision-making link of turboshaft engine around the above two schemes.

The performance demonstration composes of three sub-procedures: concept analysis of model system, service environment analysis and function analysis of model system, which used to transform the ability demanded of turboshaft engine into performance demanded. Under the ability demanded scheme, the basic behavior and function could be got by above three sub-procedures. Under the consideration of present and future industrial technology, the performance that the turboshaft engine can reach is supreme performance demanded. The ability demanded which is got on requirement demonstration is the most basic demand for turboshaft engine, as well its mapping performance is the most basic and attainable performance demanded. The balance between attainable performances with supreme performance could emerge various performance demanded schemes. Then, the evaluation and decision-making of the performance demanded schemes constitutes the performance demonstration and decision-making link of turboshaft engine.

Comprehensive model system demonstration composes of two sub-procedures: model system structure analysis and feasibility analysis, which used to draw up the structure schemes by performance demanded scheme. Different structure schemes have different materials techniques, component and component schemes. Even for the same performance scheme, there were multiple possible alternatives. Then, it need to conduct evaluation and make decision to get the optimal structure scheme, such process constitutes the structure scheme demonstration and decision-making link of turboshaft engine.

Tactical technique index demonstration composes of two sub-procedures: model system function analysis and index system analysis, which used to establish tactical technique index schemes of turboshaft engine by above performance demanded. The common and characteristic tactical technique performance items are discomposed into the most basic performance component factors on 
the model system function analysis sub-procedure. Later, the performance model is established to analyze the system properties which are mapped by each tactical technique performance. At last, it can build the tactical technique index system of turboshaft engine model system by these system performance models and each performance component factors. To reach the performance demanded of turboshaft engine, it is necessary to balance and modify these above-mentioned tactical technique indexes. So, this progress constitutes the tactical technique index demonstration and decision-making link of turboshaft engine.

\section{The Assistant Decision-making Effect of the Analysis of effectiveness and cost on the demonstration of turboshaft engine}

From above, all demonstration and decision-making links are the process of iteration and adjustment until the final decision. There are similar characteristics in these links that is the given multi-object demanded, the multifactorial influence, and they all need to balance and optimize decision in multiple alternatives. For this reason, the analysis of effectiveness and cost is a system analysis method about how to select optimal solution in a number of solutions; it could provide scientific and effective support on demonstration and decision-making process of turboshaft engine. In general, the procedure of analysis of effectiveness and cost include six factors: objective, scheme, cost, efficiency, model and decision criterion [2]. Obviously, the manifestations of these factors are different influenced by stage, extent of information on each demonstration and decision-making links. So, the assistant decision-making effect of the analysis of effectiveness and cost are different, too.

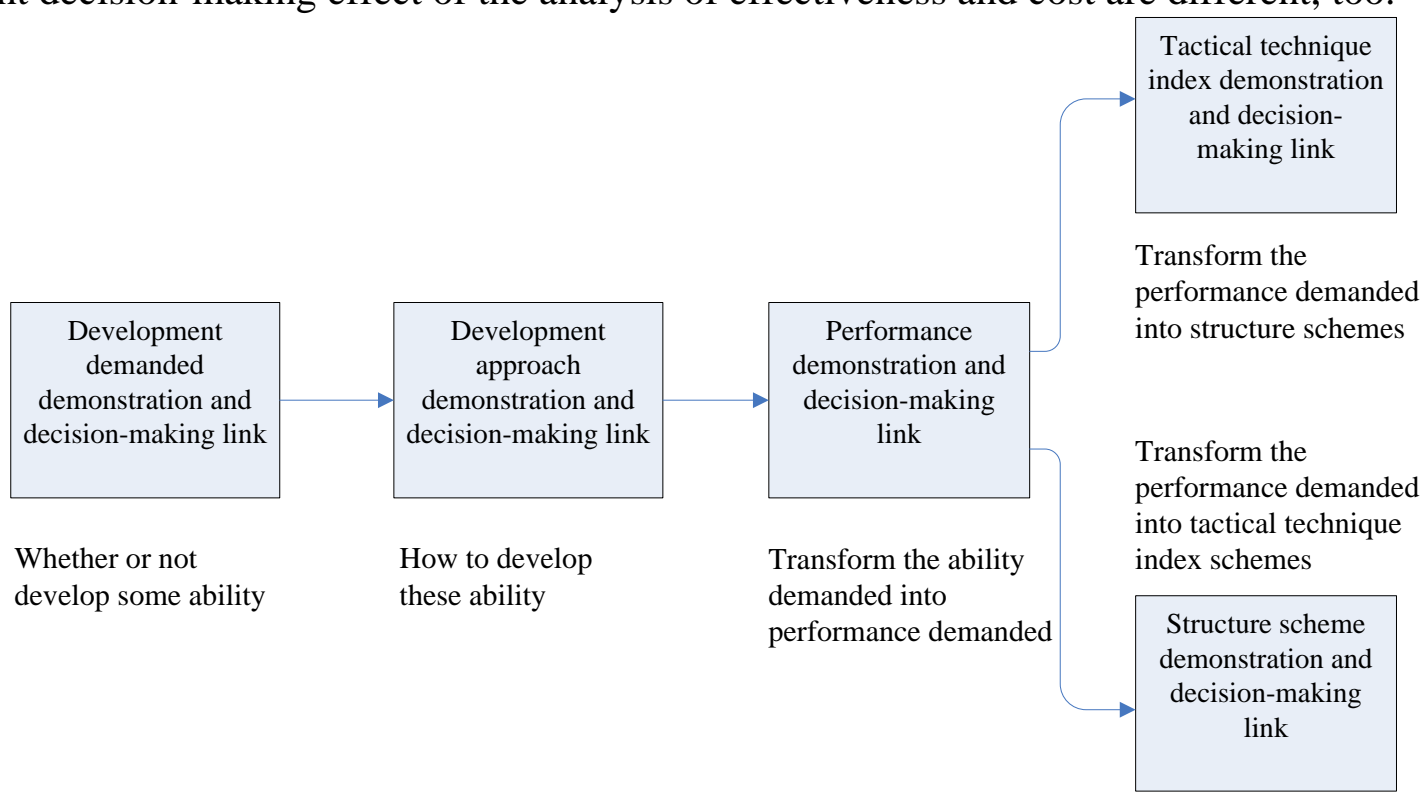

Fig. 2. Demonstration and decision-making procedure of turboshaft engine

In the development demanded demonstration and decision-making link of turboshaft engine, the objective is about developing the ability demanded of turboshaft engine that can satisfy the future usage of helicopter, and the combinations of different ability demanded build various ability demanded schemes. Later, the turboshaft engines which have current cost and technical data as standard, it can evaluate the life cycle cost by comparing the above schemes. Due to the development demanded demonstration and decision-making link is in the early of demonstration procedure of turboshaft engine, so it selects single efficiency as measure of efficiency. At last, the function of effectiveness, cost can be established with single efficiency index. For example, we can choose the power as single efficiency index, then the efficiency function is $E=a_{1} X+b_{1}$, the cost function is $\mathrm{C}=\mathrm{a}_{2} \mathrm{X}+\mathrm{b}_{2}$, where E represents efficiency, $\mathrm{C}$ represents cost, $\mathrm{X}$ represents power, and $\mathrm{a}_{1} 、 \mathrm{a}_{2}$ 、 $b_{1} 、 b_{2}$ are coefficients. With the rank of the cost-effectiveness ratio, we can select the optimal scheme. 
In the development approach demonstration and decision-making link, the objective is to confirm how to realize the ability demanded in previous link. At this moment, there are two different development schemes, one is directly improving the existing engines, another is developing new pattern turboshaft engine. In this link, the cost estimation methods, measure of efficiency and decision criterion are the same with previous link, here is no need to do.

In the performance demonstration and decision-making link, the objective is to find appropriate performance demanded by ability demanded. The balance between attainable performances with supreme performance could emerge various performance demanded schemes. Due to the earlier demonstration, we can also use analogous estimates to evaluate life cycle cost. And for the efficiency, we generally use gray analytic hierarchy process [3]. For example, we can get the hierarchy of performance, as shown in figure 3; secondly, the combination weight of the underlying performance $\mathrm{W}=\left(\omega_{1}, \omega_{2}, \ldots, \omega_{12}\right)^{\mathrm{T}}$ is got by expert judgment; later, we could get the evaluation index matrix $D_{i j}^{\left({ }^{(A)}\right.}=\left[\begin{array}{cccc}d_{11}^{(A)} & d_{12}^{(A)} & \cdots & d_{1 i}^{(A)} \\ d_{21}^{(A)} & d_{22}^{(A)} & \cdots & d_{2 i}^{(A)} \\ \vdots & \vdots & \ddots & \vdots \\ d_{j 1}^{(A)} & r_{j 2}^{(A)} & \cdots & r_{j i}^{(A)}\end{array}\right]$, where $\mathrm{D}_{\mathrm{JI}}^{(\mathrm{A})}$ represents the evaluation index matrix is made by the evaluator I to the ratee $\mathrm{J}$ about the Ath evaluation factors; Then, we should determine the gray class evaluation, the gray evaluation coefficient $\mathrm{n}_{\mathrm{JK}}^{(\mathrm{A})}$, the total gray evaluation coefficient $n_{J}^{(A)}=\sum_{i=1}^{k} n_{J i}^{(A)}$, the gray evaluation weight vectors $r_{J K}^{(A)}=\frac{n_{J K}^{(A)}}{n_{J}^{(A)}}$ and the gray evaluation weight matrix $R^{(A)}=\left[\begin{array}{cccc}r_{11}^{(A)} & r_{12}^{(A)} & \cdots & r_{1 k}^{(A)} \\ r_{21}^{(A)} & r_{22}^{(A)} & \cdots & r_{2 k}^{(A)} \\ \vdots & \vdots & \ddots & \vdots \\ r_{j 1}^{(A)} & r_{j 2}^{(A)} & \cdots & r_{j k}^{(A)}\end{array}\right)$; finally, the index evaluation weight vectors $r^{*(A)}=\left\{r_{1}^{*(A)}, r_{2}^{*(A)}, \ldots, r_{j}^{*(A)}\right\}$ are got by the evaluation of each evaluation indexes, and the schemes can be in order by $\mathrm{r}^{*}{ }^{(\mathrm{A})}$.

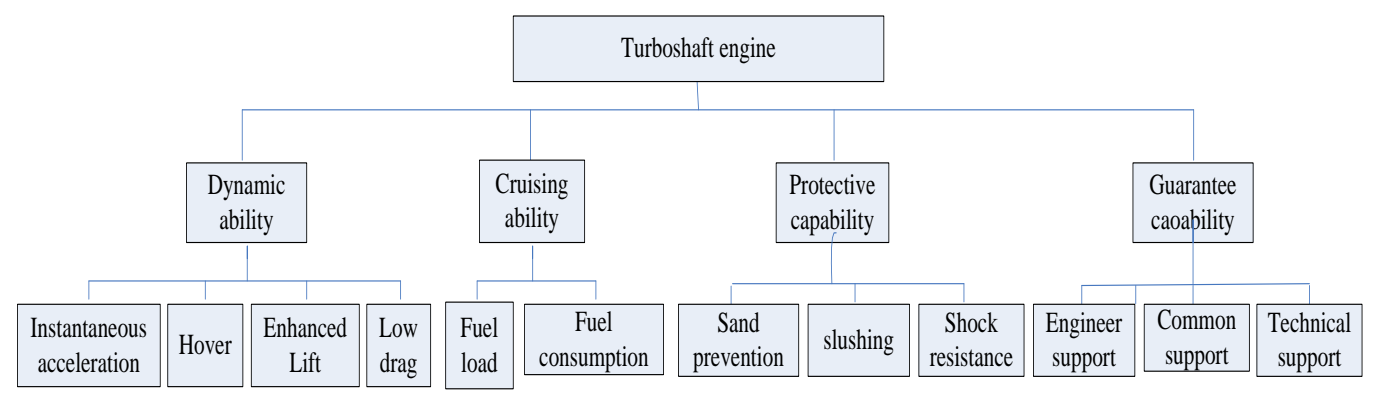

Fig. 3. Performance hierarchy

In the structure scheme demonstration and decision-making link, the objective is to draw up appropriate structure scheme by performance demanded. At this moment, the material selection, present industrial level, and development time will have an effect on specific structure scheme, namely produce alternative structure schemes. In this link, we should transform the technical advancement, development risk and time into cost. At present, it selects system efficiency as measure of efficiency. With the function $\mathrm{E}=\mathrm{A} \bullet \mathrm{D} \bullet \mathrm{C}$, the efficiency will be evaluated. At last, base on the equal efficiency of each scheme, the optimal one will be chosen by its lowest cost.

In the tactical technique index demonstration and decision-making link, the object is to draw up appropriate tactical technique index scheme by performance demanded. Indexes should be balanced and adjusted to satisfy the performance demanded that it constitute various tactical technique index schemes. With the hierarchy of cost, as shown in figure 3; then, the life cycle cost of turboshaft 
engine is: $L C C=C_{1}+C_{2}+C_{3}+C_{4}+C_{5}$, where $C_{1}$ represents the demonstration cost, $C_{2}$ represents the development cost, $\mathrm{C}_{3}$ represents the purchase cost, $\mathrm{C}_{4}$ represents the use and logistical cost, and $\mathrm{C}_{5}$ represents the retired cost; finally, we can get the life cycle cost by parametric estimating and specific formulas for calculating indexes. Due to this link is in the later period of demonstration procedure of turboshaft engine, so it selects the operational efficiency as measure of efficiency. Generally, the index method is used to evaluate the operational efficiency. The index computational formula is $E_{i}=D+C+P+G$, where $D$ is dynamic ability, $C$ is cruising index, $P$ is protective index, $G$ is guarantee index. Finally, consider the complexity, sensitiveness and so on of the tactical technique indexes, the optimal scheme is chosen by the efficiency-cost increasing standard.

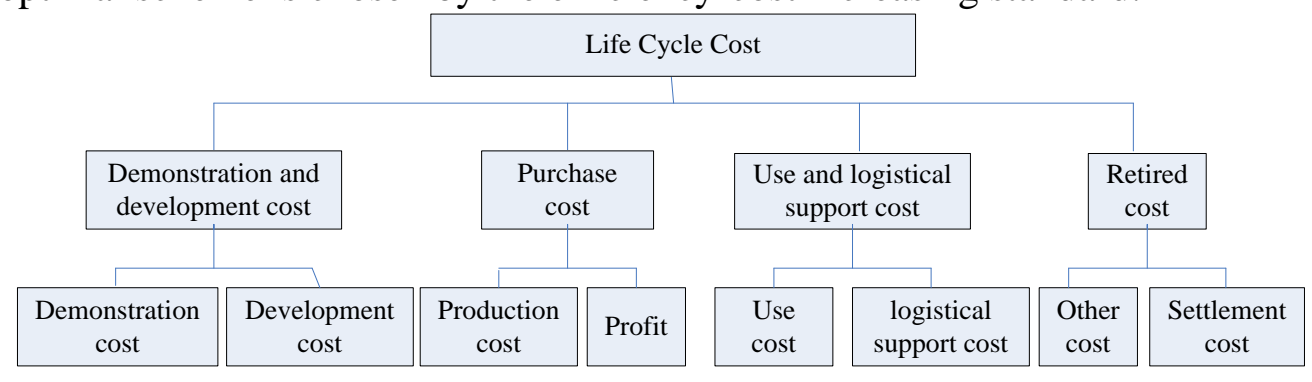

Fig. 4. Cost hierarchy

\section{Summary}

This article systematically expounds the demonstration and decision-making process of turboshaft engine, reveals the various demanded for multi-objective, multi-factor and multi-scheme decision-making in different links, proposes the effects, principles and methods of the analysis of effectiveness and cost in each demonstration and decision-making link. But the article is still a beginning in related research, it could make further research in effectiveness-cost models, uncertainty processing and sensitivity analysis in the future.

\section{References}

[1] Ming L, Peng L. Theory and practice of an integrated and systematic demonstration method for development of weapon and equipment [M]. National Defense Industry Press (Beijing), 2000.

[2] Yuquan W. The cost-efficiency analysis of equipment[M]. National Defense Industry Press (Beijing), 2010.

[3] Qisheng G. Introduction to equipment effectiveness evaluation [M]. National Defense Industry Press (Beijing), 2005.

[4] Luxiang L. The model development project of military helicopter[M]. National Defense Industry Press (Beijing), 2010.

[5] Hongtao X, Haitao Z. Comprehensive analysis of the cost-effectiveness for civil helicopters [J]. Aeronautical Science and Technology, 2014(1):62-68.

[6] Haitao Z. Helicopter cost estimating based on the empty weight [J]. Aeronautical Science and Technology, 2013(2):18-21. 